\title{
Stellar Distance and Velocity (II)
}

\author{
Milos Cojanovic \\ Independent Scholar Montreal, Canada Email: cojmilmo[at]gmail.com \\ September 3, 2019
}

\begin{abstract}
In this paper we will point out the systematic error, which is made using the parallax in determining the stellar distance. Instead of this method, we are proposing three methods that give more precise results in calculating the distance. In the first method we will use two consecutive parallaxes instead of one, which will allow us to determine the distance of the stars with negative parallaxes. In the second one we will present a method by which it is possible to measure a true parallax. The third method is already presented in [1] and here we will consider only one special case when the velocity of a star is calculated relative to the sun. We tested the methods using data obtained from the Gaia's catalogs [2].
\end{abstract}

Keywords: stellar parallax, stellar distance, stellar velocity

\section{Introduction}

Stellar parallax is defined as the apparent change in angular position of a particular star against more distant background stars using two points in the earth's orbit around the sun as the baseline. The stellar parallax obtained in this way can be called "observed stellar parallax". It is assumed that the position of the observed star within a period of six months is fixed regarding to the sun. Therefore, the parallax is caused only by the movement of the earth about the sun Figure (1).

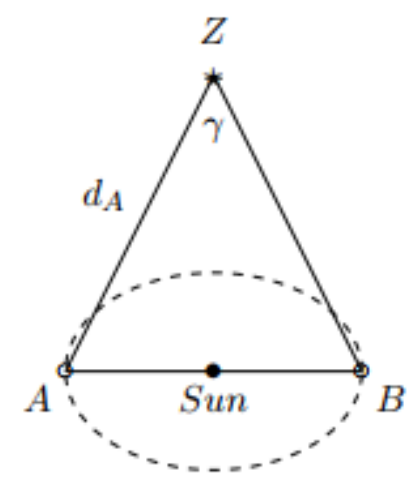

Figure 1: A generally accepted viewpoint according to which the position of the star regarding the sun remains unchanged for six months.

The reason why it is usually assumed that a star position remains fixed relative to the sun, is that the star is so far away thus a change in its position does not significantly affect the measurement of parallax. Which is not true. In some cases, the change in the position of the star in relation to the sun affects the parallax as much as the change in the position of the earth. Let us consider now, the situation given in Figure (2). Suppose that the star (Z) moves in the direction of $\mathrm{AB}$.

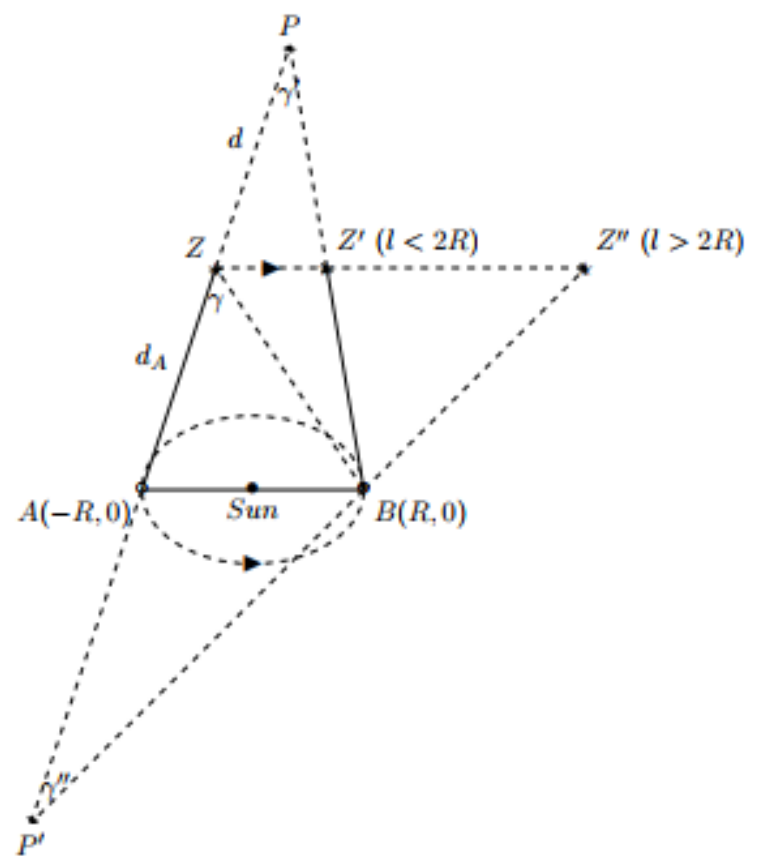

Figure 2: A star is changing its position regarding the sun

Unlike the situation shown in Figure (1), the position of a star has been changed from the point $Z$ to the point $Z^{\prime}\left(Z^{\prime \prime}\right)$.

Let AZ, AP and AP' are defined as follows:

1) $\mathrm{AZ}=$ true distance between the Earth and a star at some moment $\left(\mathrm{t}_{0}\right)$

2) $\mathrm{AP}=$ distance derived from the parallax $\gamma^{\prime}$ six months later (parallax $>0$ ).

3) $\mathrm{AP}^{\prime}=$ distance derived from the parallax $\gamma$ ' six months later (parallax <0).

- $\mathrm{R}=\mathrm{AU}=149597870.7[\mathrm{~km}]$

- year sec $=365.25 * 24 * 3600[\mathrm{sec}]$

If $(\Pi-(\alpha+\beta)>0)$ then we will have the following definitions and equations:

$$
\begin{array}{r}
A B \| Z Z^{\prime} \\
d_{A}=A Z \\
d=A P \\
l=Z Z^{\prime} \\
\alpha=\angle(B, A, Z)
\end{array}
$$

Volume 8 Issue 9, September 2019

www.ijsr.net

Licensed Under Creative Commons Attribution CC BY 


\section{International Journal of Science and Research (IJSR)}

ISSN: 2319-7064

ResearchGate Impact Factor (2018): 0.28 | SJIF (2018): 7.426

$$
\begin{array}{r}
\beta=\angle\left(Z^{\prime}, B, A\right) \\
\gamma^{\prime}=\Pi-(\alpha+\beta)>0 \\
\frac{d}{d-d_{A}}=\frac{2 R}{l} \\
\frac{d}{d_{A}}=\frac{2 R}{2 R-l}
\end{array}
$$

If $(\Pi-(\alpha+\beta)<0)$ then we will have following equations:

$$
\begin{array}{r}
d=A P^{\prime} \\
l=Z Z^{\prime \prime} \\
\beta=\angle\left(Z^{\prime \prime}, B, A\right) \\
\gamma^{\prime \prime}=(\alpha+\beta)-\Pi \\
\frac{d+d_{A}}{d}=\frac{l}{2 R} \\
\frac{d}{d_{A}}=\frac{2 R}{l-2 R}
\end{array}
$$

The Equation (9) can be written in a different way:

$$
\begin{array}{r}
\Delta t=t_{1}-t_{0}=0.5 * \text { yearsec } \\
\frac{R * \pi}{\Delta t} \approx 30[\mathrm{~km} / \mathrm{sec}] \\
2 R=\frac{2 * 30 * \Delta t}{\pi} \approx 19.1 * \Delta t \\
l=\Delta u_{x} * \Delta t \\
\frac{l}{2 R} \approx \frac{\Delta u_{x} * \Delta t}{19.1 * \Delta t} \approx \frac{\Delta u_{x}}{19.1} \\
\frac{d}{d_{A}} \approx \frac{19.1}{19.1-\Delta u_{x}}
\end{array}
$$

where $\Delta \mathrm{u}_{\mathrm{x}}$ denotes star velocity in the direction $\mathrm{AB}$.

For example, when in Equation (21) we substitute $\Delta \mathrm{u}_{\mathrm{x}}$ for (10) it follows that calculated distance $d$ is about times greater than true distance $d_{A}$ or in case when we substitute $\Delta \mathrm{u}_{\mathrm{x}}$ for $(-10)$ we've got that calculated distance $\mathrm{d}$ is about 0.65 times less than true distance $d_{A}$.

By analyzing the Equation (9) it follows that there are four possibilities:

1) if $(1=0) \Rightarrow\left(d=d_{A}\right)$ (distance $d$ is equal to the true distance $d_{A}$ )

2) if $(1>2 \mathrm{R}) \Rightarrow(\alpha+\beta>\Pi) \Rightarrow\left(\gamma^{\prime}=\Pi-(\alpha+\beta)<0\right) \Rightarrow$ (derived distance $\mathrm{d}$ is negative)

$3)$ if $(0<1<2 R) \Rightarrow$ derived distance $d$ is greater than the true distance $\mathrm{d}_{\mathrm{A}}$

4) if $(1<0) \Rightarrow$ derived distance $d$ is less than the true distance $d_{A}$

From the Equation (21) it follows that the error we make by calculating the distance $\mathrm{d}$ does not depend only on the true distance $\mathrm{d}_{\mathrm{A}}$ but also from the relative velocity $\Delta \mathbf{u}$ at which the star moves in relation to the sun (actually, mostly due to its component $\Delta \mathrm{u}_{\mathrm{x}}$, since the other two components $\Delta \mathrm{u}_{\mathrm{y}}$ and $\Delta \mathrm{u}_{\mathrm{y}}$ are left out from consideration)). The best result in calculating the distance we have in the case when the $\Delta \mathrm{u}_{\mathrm{x}}=$ 0 , in other words when the diameter $\mathrm{AB}$ is the perpendicular to the projection of the velocity $\Delta \mathbf{u}$ regarding the ecliptic plane $\left(\mathrm{O}_{\mathrm{xy}}\right)$. But if the velocity $\Delta \mathbf{u}$ is known, it implies that in addition to knowing a proper motion, it must also be known a distance $\mathrm{d}_{\mathrm{A}}$, what we are actually looking for.
On the basis of these analyzes, it can be concluded that the use of observed parallax in determining the stellar distances should be used with great caution.

\section{Finding stellar distance by measuring two consecutive parallax angles $(2 \mathrm{CP})$}

In the previous section, it has been proved that by applying the parallax method in the finding of the distance we make a certain systematic error. In this section we are presenting a method in which two successive parallax measurements are used in determining the distance. In this case there are also some systematic errors, but they are far less compared to the standard parallax method.

We are assuming that star is moving uniformly by velocity $\Delta \mathbf{u}=\left[\Delta \mathrm{u}_{\mathrm{x}}, \Delta \mathrm{u}_{\mathrm{y}}, \Delta \mathrm{u}_{\mathrm{z}}\right]$ regarding the sun. Its component noted by $\Delta \mathrm{u}_{\mathrm{x}}$ is parallel to the arbitrarily chosen diameter $\mathrm{AB}$ Figure (3).

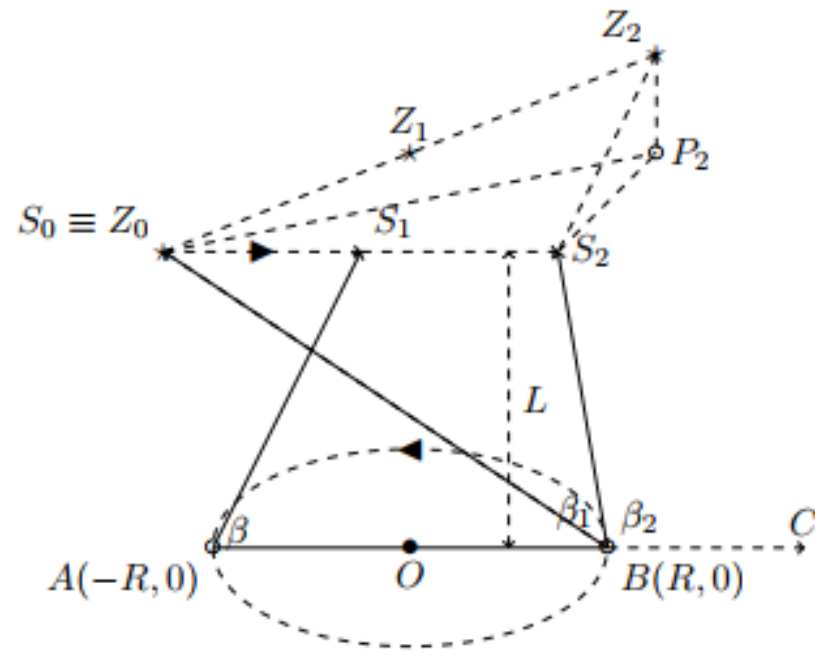

Figure 3: Determining the distance by measuring two successive parallax angles

At a some moment $t_{0}$ the position of the Earth is indicated by the point $\mathrm{B}$ and the position of a star is shown by the point $\mathrm{Z}_{0}=\left(\lambda_{1}, \beta_{1}\right)$. After six (eighteen, thirty, ..) months later Earth will reach the point $A$ and star will reach the point $Z_{1}=(\lambda$, $\beta)$. And again, six (eighteen, thirty, ..) months later Earth will reach the point $\mathrm{B}$ and star will reach the point $\mathrm{Z}_{2}=\left(\lambda_{2}\right.$, $\beta_{2}$ ). The first component denotes ecliptic longitude and the second denotes ecliptic latitude of the star. We will now assume that direction $\mathrm{AB}$ approximately coincides with the star's ecliptic longitude $\lambda_{1}$. Latitudes with negative values will be treated as having positive values.

Referring to Figure (3) we have the following relations and equations:

$$
\begin{array}{r}
\Delta t=(0.5+m) * \text { yearsec } \\
\text { where }\{m=0,1,2,3 . .\} \\
S_{0} S_{2} \| A B \\
Z_{0} Z_{1}=Z_{1} Z_{2}=\Delta u * \Delta t \\
S_{0} S_{1}=S_{1} S_{2}=u_{x} * \Delta t \\
\beta_{1}=\angle\left(C, B, S_{0}\right)=\angle\left(C, B, Z_{0}\right) \\
\beta=\angle\left(C, A, Z_{1}\right)
\end{array}
$$

\section{Volume 8 Issue 9, September 2019}

\section{www.ijsr.net}




\section{International Journal of Science and Research (IJSR)}

ISSN: 2319-7064

ResearchGate Impact Factor (2018): 0.28 | SJIF (2018): 7.426

$$
\begin{array}{r}
\angle\left(C, A, S_{1}\right)=\beta \\
\beta_{2}=\angle\left(C, B, Z_{2}\right) \\
\angle\left(C, B, S_{2}\right)=\beta_{2} \\
S_{0}=\left(R+L * \frac{\cos \left(\beta_{1}\right)}{\sin \left(\beta_{1}\right)}, L\right) \\
S_{2}=\left(R+L * \frac{\cos \left(\beta_{2}\right)}{\sin \left(\beta_{2}\right)}, L\right) \\
S_{1}=\left(-R+L * \frac{\cos (\beta)}{\sin (\beta)}, L\right) \\
\left(-R+L * \frac{\cos (\beta)}{\sin (\beta)}, L\right)=\left(R+L * \frac{\cos \left(\beta_{1}\right) * \sin \left(\beta_{2}\right)+\cos \left(\beta_{2}\right) * \sin \left(\beta_{1}\right)}{2 * \sin \left(\beta_{1}\right) * \sin \left(\beta_{2}\right)}, L\right)= \\
\left(R+L * \frac{\sin \left(\beta_{2}+\beta_{1}\right)}{2 * \sin \left(\beta_{1}\right) * \sin \left(\beta_{2}\right)}, L\right) \\
B S_{0}=\frac{\left(\beta_{2}\right.}{\sin \left(\beta_{1}\right)}=4 R * \frac{\sin \left(\beta_{1}+\beta_{2}\right) * \sin (\beta)}{2 * \sin \left(\beta_{1}\right) * \sin \left(\beta_{2}\right) * \cos (\beta)-\sin \left(\beta_{1}+\beta_{2}\right) * \sin (\beta)} \\
L=4 * \frac{2 * \sin \left(\beta_{1}\right) * \sin \left(\beta_{2}\right) * \cos (\beta)-\sin \left(\beta_{2}\right) * \sin (\beta)}{2 * \sin \left(\beta_{1}\right) * \sin \left(\beta_{2}\right) * \sin (\beta)}
\end{array}
$$

If we denote $B$ by $B(-R ; 0)$ instead of $B(R ; 0)$ and denote $A$ by $A(R ; 0)$ instead of $A(-R ; 0)$ Figure (3), we get the same result as in Equation (40) but with opposite sign. Equation (40) can be written as follows:

$$
B S_{0}=4 R * \frac{\sin \left(\beta_{2}\right) * \sin (\beta)}{\left|2 * \sin \left(\beta_{1}\right) * \sin \left(\beta_{2}\right) * \cos (\beta)-\sin \left(\beta_{1}+\beta_{2}\right) * \sin (\beta)\right|}
$$

By using this method we also made several systematic errors. First, components $\mathrm{u}_{\mathrm{y}}$ and $\mathrm{u}_{\mathrm{z}}$ were left out from calculations. This means that the actual positions of the star marked by $Z_{1}, Z_{2}$ are respectively replaced by $S_{1}, S_{2}$. Then, the time it takes for the star to move from point $Z_{0}$ to point $Z_{1}$ and from point $Z_{1}$ to point $Z_{2}$ is not equal to $\Delta t$.

Referring to Figure (3) we have following definitions and equations:

$$
\begin{array}{r}
\Delta t_{2}=2 * \Delta t \\
\delta=\angle\left(P_{2}, Z_{0}, Z_{2}\right) \\
\theta=\angle\left(S_{2}, Z_{0}, P_{2}\right) \\
S_{2} P_{2}=\Delta t_{2} * u_{y} \\
P_{2} Z_{2}=\Delta t_{2} * u_{z} \\
Z_{0} S_{2}=\Delta t_{2} * u_{x}=\Delta t_{2} * \Delta * \cos (\delta) * \cos (\theta) \\
Z_{0} Z_{2}=\Delta t_{2} * \Delta u \\
\sqrt{\left(Z_{0} Z_{2}\right)^{2}-\left(Z_{0} S_{2}\right)^{2}}=\Delta t_{2} * \Delta u * \sqrt{1-(\cos (\delta) * \cos (\theta))^{2}}(
\end{array}
$$

It is obvious that the point $S_{2}$ is closer to the point $Z_{2}$, the more accurate the result will be. For a given vector $\Delta \mathbf{u}$, the angle $\delta$ that lies between the ecliptic plane $\left(\mathrm{O}_{\mathrm{xy}}\right)$ and the vector $\Delta \mathbf{u}$ is fixed. Therefore, it follows from the Equation (49) that the $S_{2} Z_{2}$ has a minimum length if $\theta=0$, in other words when the parallax angle has approximately the maximum value.

The advantage of this method is that it is relatively easy to use, also the measurements can be performed over a period of time. In this way, the parallax angles are increasing, but the drawback is that systematic errors are also accumulating.

Obviously, we did not directly use the parallax angle, but we associated the method name with the parallax angle primarily to make this method easier to distinguish from other methods.

Now we will present two methods that do not have systematic errors.

\section{Measuring a true stellar parallax}

We are assuming that all the positions and angles are referred to the International Celestial Reference Frame (currently ICRF3), extragalactic reference frame of the ICRS (the International Celestial Reference Frame). The ICRF creates a quasi-inertial frame of reference centered at the barycenter of the Solar System, whose axes are defined by the measured positions of extragalactic sources (mainly quasars)[3]. For simplicity, the coordinate system will be marked by $(\mathrm{K})$.

Our idea is very simple. Instead of having two measurements from a single location within six months, we will simultaneously perform two measurements from two different locations. It would practically mean that there should be two satellites that would move in the vicinity of the two planets from the solar system. In addition, each satellite should have one clock, so both clocks show the

\section{Volume 8 Issue 9, September 2019 www.ijsr.net}




\section{International Journal of Science and Research (IJSR) \\ ISSN: 2319-7064}

ResearchGate Impact Factor (2018): 0.28 | SJIF (2018): 7.426

same time. We will not deal with further technical details, except that we will conclude that such an experiment in view of the level reached by the current technology is possible.

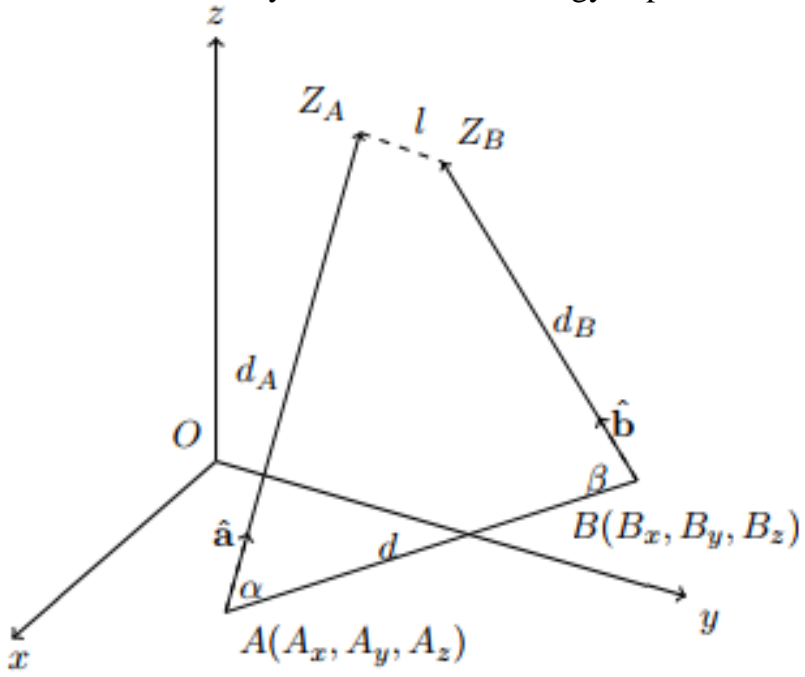

Figure 4: The point A denotes the position of the first and the point $\mathrm{B}$ denotes position of the second satellite at the some instant $\mathrm{t}$

It is assumed that during the measurement we made random errors, and hence the star position at the instant $t$ is shown by two different points marked by ZA and ZA.

Referring to Figure (4) we can define the following parameters:

- $\mathrm{d}$ - distance between two satellites at the some instant $\mathrm{t}$

- $\mathrm{d}_{\mathrm{A}}$ - distance between the first satellite and a star

- $\mathrm{d}_{\mathrm{B}}$ - distance between the second satellite and a star

- $\mathbf{a}=\left[\mathrm{a}_{\mathrm{x}}, \mathrm{a}_{\mathrm{y}}, \mathrm{a}_{\mathrm{z}}\right]$ - unit vector determined by the positions of the first satellite and a star regarding the $(\mathrm{K})$

- $\mathbf{b}=\left[b_{\mathrm{x}}, \mathrm{b}_{\mathrm{y}}, \mathrm{b}_{\mathrm{z}}\right]$ - unit vector determined by the positions of the second satellite and a star regarding the $(\mathrm{K})$

$$
\begin{array}{r}
\mathbf{A B}=\left[B_{x}-A_{x}, B_{y}-A_{y}, B_{z}-A_{z}\right] \\
d=\mid \mathbf{A B}=\sqrt{\left(B_{x}-A_{x}\right)^{2}+\left(B_{y}-A_{y}\right)^{2}+\left(B_{z}-A_{z}\right)^{2}} \\
\mathbf{A B} \cdot \mathbf{a}=d * 1 * \cos \alpha \\
\cos \alpha=\frac{\mathbf{A B} \cdot \mathbf{a}}{d} \\
\mathbf{b} \cdot \mathbf{B A}=d * 1 * \cos \beta \\
\cos \beta=\frac{\mathbf{b} \cdot \mathbf{B A}}{d}
\end{array}
$$

Now we can construct a triangle $\triangle \mathrm{ABZ}$ Figure (5) whose one side is equal to $d$, and has two angles of $\alpha$ and $\beta$. If $\alpha+\beta$ $\geq \Pi$ then it is obvious that the triangle $\triangle \mathrm{ABZ}$ cannot be constructed. The reason why this could happen was because a random or systematic error has been made or the object is too far away.

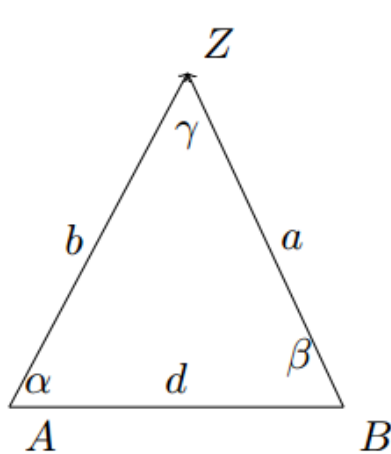

Figure 5: The two satellites and a star are forming triangle $\triangle \mathrm{ABZ}$

Referring to Figure (5) we have following equations:

$$
\begin{array}{r}
\gamma=\Pi-(\alpha+\beta) \\
\frac{b}{\sin (\beta)}=\frac{a}{\sin (\alpha)}=\frac{d}{\sin (\gamma)} \\
a=\frac{d * \sin (\alpha)}{\sin (\gamma)} \\
b=\frac{d * \sin (\beta)}{\sin (\gamma)}
\end{array}
$$

Referring to Figure (4) it follows that

$$
\begin{aligned}
& d_{A}=a \\
& d_{B}=b
\end{aligned}
$$$$
\mathbf{A} \mathbf{Z}_{A}=\left[A_{x}+d_{A} * a_{x}, A_{y}+d_{A} * a_{y}, A_{z}+d_{A} * a_{z}\right]
$$$$
\mathbf{B Z}_{B}=\left[B_{x}+d_{B} * b_{x}, B_{y}+d_{B} * b_{y}, B_{z}+d_{B} * b_{z}\right]
$$$$
\mathbf{Z}_{A} \mathbf{Z}_{B}=\mathbf{Z}_{A} \mathbf{A}+\mathbf{A B}+\mathbf{B} \mathbf{Z}_{B}
$$$$
l=\left|\mathbf{Z}_{A} \mathbf{Z}_{B}\right|
$$

if $(1>\mathrm{L})$ where $\mathrm{L}$ denotes some predetermined positive number, we will say that the experiment failed.

$$
\mathbf{O Z}=\frac{\mathbf{O Z}_{A}+\mathbf{O Z} \mathbf{Z}_{B}}{2}
$$

Otherwise we can define a point $\mathrm{Z}$ in the following way:

Suppose we performed $N$ such experiments Figure (6). Let $t_{i}$ denote the time and $d_{i}$ denotes the distance between the sun and the star in the $\mathrm{i}^{\text {th }}$ experiment.

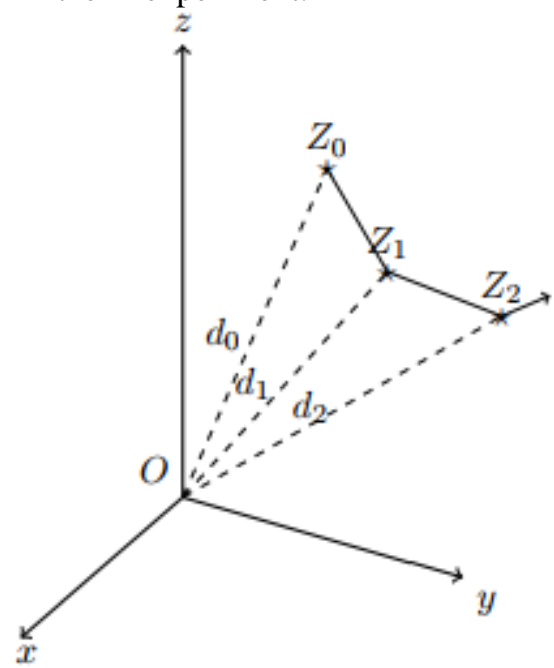

Figure 6: Repeating the same procedure it is possible to determine a trajectory of the star then we can define $\tau i$ as the time when the light is emitted from the star in this way. 


\section{International Journal of Science and Research (IJSR) \\ ISSN: 2319-7064}

ResearchGate Impact Factor (2018): 0.28 | SJIF (2018): 7.426

Then we can define $\tau_{i}$ as the time when the light is emitted from the star in this way.

$$
\tau_{i}=t_{i}-\frac{d_{i}}{c}
$$

where $\mathrm{c}$ as usual represents constant speed of the light

Then we have the following equations:

$$
\begin{gathered}
\Delta \tau_{i}=\tau_{i}-\tau_{i-1}=\left(t_{i}-\frac{d_{i}}{c}\right)-\left(t_{i-1}-\frac{d_{i-1}}{c}\right)=\left(t_{i}-t_{i-1}\right) \\
-\frac{d_{i}-d_{i-1}}{c}=\Delta t_{i}-\frac{\Delta d_{i}}{c}
\end{gathered}
$$

The average velocity $\Delta \mathbf{u}_{\mathrm{i}}$ of a star relative to sun from point $\mathrm{Z}_{\mathrm{i}}-1$ to point $\mathrm{Z}_{\mathrm{i}}$ can be defined as

$$
\Delta \mathbf{u}_{i}=\frac{\mathbf{Z}_{i-1} \mathbf{Z}_{i}}{\Delta \tau_{i}}
$$

In this way we can determine a trajectory Figure (6) of the star and its velocity relative to the sun.

The proposed experiment is theoretically possible, but the question is whether it will ever be realized. Therefore, in the next section, we will briefly and as simple as possible describe the method already presented in [1]. Some of the formulas will be given in a different form.

\section{Determining the stellar distance and velocity (3P)}

In this case, instead of two measurements it is necessary to make three measurements. We assume that a star is moving with a uniform, rectilinear space motion regarding the sun.

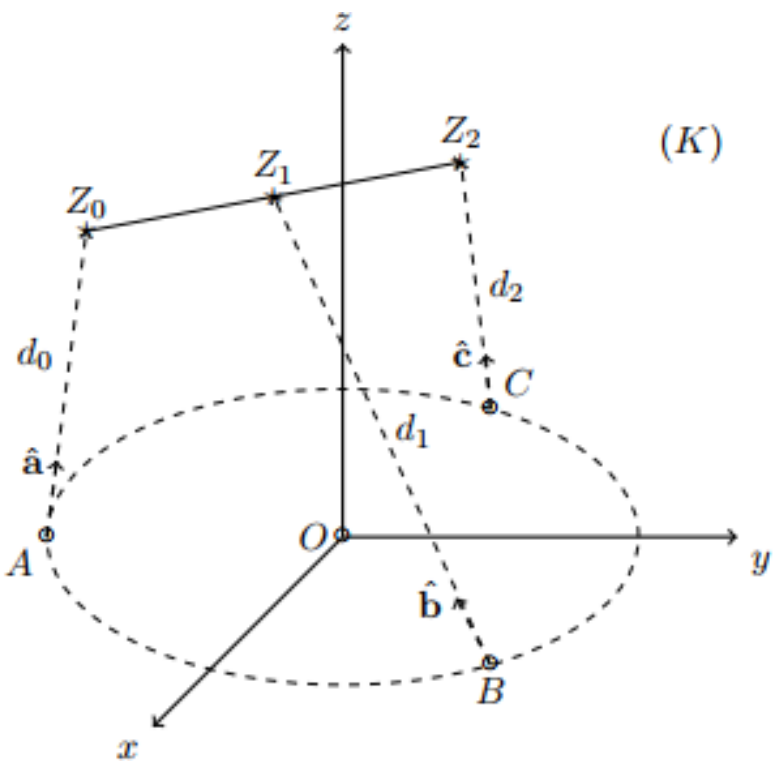

Figure 7: Points A, B, C do not lie on the same line. There is one nontrivial solution.

The following fields Figure (7) were obtained by direct measurements:

- $A=\left(A_{x}, A_{y}, A_{z}\right)-$ denotes the position of the Earth (satellite) at the time $\left(\mathrm{t}_{0}\right)$

- $\hat{\mathbf{a}}^{\hat{}}=\left[\mathrm{a}_{\mathrm{x}}, \mathrm{a}_{\mathrm{y}}, \mathrm{a}_{\mathrm{z}}\right]$ - denotes the unit vector determined by the point $A$ and position of the star $\left(Z_{0}\right)$

- $\mathrm{B}=\left(\mathrm{B}_{\mathrm{x}}, \mathrm{B}_{\mathrm{y}}, \mathrm{B}_{\mathrm{z}}\right)$ - denotes the position of the Earth (satellite) at the time $\left(t_{1}\right)$
- $\mathbf{b}^{\wedge}=\left[b_{\mathrm{x}}, b_{\mathrm{y}}, \mathrm{b}_{\mathrm{z}}\right]$ - denotes the unit vector determined by the point $\mathrm{B}$ and position of the $\operatorname{star}\left(\mathrm{Z}_{1}\right)$

- $\mathrm{C}=\left(\mathrm{C}_{\mathrm{x}}, \mathrm{C}_{\mathrm{y}}, \mathrm{C}_{\mathrm{z}}\right)$ - denotes the position of the Earth (satellite) at the time $\left(\mathrm{t}_{2}\right)$

- $\mathbf{c}^{\wedge}=\left[\mathrm{c}_{\mathrm{x}}, \mathrm{c}_{\mathrm{y}}, \mathrm{c}_{\mathrm{z}}\right]-$ denotes the unit vector determined by the point $\mathrm{C}$ and position of the $\operatorname{star}\left(\mathrm{Z}_{2}\right)$

The time interval between the measurements is arbitrary. Knowing all of the above fields we are able to calculate the following values:

$$
\begin{aligned}
& \Delta t_{1}=t_{1}-t_{0} \\
& \Delta t_{2}=t_{2}-t_{0}
\end{aligned}
$$

$$
\begin{aligned}
M & =\left[\begin{array}{cccc}
\Delta t_{2} * c & 1 & 1 & 1 \\
C_{x}-A_{x} & a_{x} & b_{x} & c_{x} \\
C_{y}-A_{y} & a_{y} & b_{y} & c_{y} \\
C_{z}-A_{z} & a_{z} & b_{z} & c_{z}
\end{array}\right] \\
N & =\left[\begin{array}{cccc}
\Delta t_{1} * c & 1 & 1 & 1 \\
B_{x}-A_{x} & a_{x} & b_{x} & c_{x} \\
B_{y}-A_{y} & a_{y} & b_{y} & c_{y} \\
B_{z}-A_{z} & a_{z} & b_{z} & c_{z}
\end{array}\right]
\end{aligned}
$$

if we define $\mathrm{k}$ as

$$
k=\frac{\overline{Z_{0} Z_{2}}}{\overline{\overline{Z_{0} Z_{1}}}}
$$

then we have that

$$
k=\frac{\operatorname{det}(M)}{\operatorname{det}(N)}
$$

We can define matrices $D ; D_{0} ; D_{1} ; D_{2}$ in following way:

$$
\begin{gathered}
D=\left[\begin{array}{lll}
(k-1) * a_{x} & -k * b_{x} & c_{x} \\
(k-1) * a_{y} & -k * b_{y} & c_{y} \\
(k-1) * a_{z} & -k * b_{z} & c_{z}
\end{array}\right] \quad(78) \\
D_{0}=\left[\begin{array}{lll}
(1-k) * A_{x}+k * B_{x}-C_{x} & -k * b_{x} & c_{x} \\
(1-k) * A_{y}+k * B_{y}-C_{y} & -k * b_{y} & c_{y} \\
(1-k) * A_{z}+k * B_{z}-C_{z} & -k * b_{z} & c_{z}
\end{array}\right](79) \\
D_{1}=\left[\begin{array}{lll}
(k-1) * a_{x} & (1-k) * A_{x}+k * B_{x}-C_{x} & c_{x} \\
(k-1) * a_{y} & (1-k) * A_{y}+k * B_{y}-C_{y} & c_{y} \\
(k-1) * a_{z} & (1-k) * A_{z}+k * B_{z}-C_{z} & c_{z}
\end{array}\right](80) \\
D_{2}=\left[\begin{array}{lll}
(k-1) * a_{x}-k * b_{x} & (1-k) * A_{x}+k * B_{x}-C_{x} \\
(k-1) * a_{y}-k * b_{y} & (1-k) * A_{y}+k * B_{y}-C_{y} \\
(k-1) * a_{z}-k * b_{z} & (1-k) * A_{z}+k * B_{z}-C_{z}
\end{array}\right](81)
\end{gathered}
$$

Now we are able to calculate the distances $d_{0}, d_{1}, d_{2}$ :

$$
\begin{aligned}
d_{0} & =\frac{\operatorname{det}\left(D_{0}\right)}{\operatorname{det}(D)} \\
d_{1} & =\frac{\operatorname{det}\left(D_{1}\right)}{\operatorname{det}(D)} \\
d_{2} & =\frac{\operatorname{det}\left(D_{2}\right)}{\operatorname{det}(D)} \\
\Delta d_{2} & =d_{2}-d_{0}
\end{aligned}
$$

\section{Volume 8 Issue 9, September 2019 www.ijsr.net}




\section{International Journal of Science and Research (IJSR) \\ ISSN: 2319-7064}

ResearchGate Impact Factor (2018): 0.28 | SJIF (2018): 7.426

$$
\begin{array}{r}
\Delta \tau_{2}=t_{2}-t_{0}-\left(\frac{d_{2}}{c}-\frac{d_{1}}{c}\right)=\Delta t_{2}-\frac{\Delta d_{2}}{c} \\
\Delta \mathbf{u}=\frac{\mathbf{Z}_{0} \mathbf{Z}_{2}}{\Delta \tau_{2}}
\end{array}
$$

where

- $\mathrm{d}_{0}$ - denotes distance between the Earth (satellite) and a star at the time (t0)

- $\mathrm{d}_{1}$ - denotes distance between the Earth (satellite) and a star at the time (t1)

- $\mathrm{d}_{2}$ - denotes distance between the Earth (satellite) and a star at the time (t2)

- $\Delta \tau_{2}$ denotes the time it takes for the star to move from point $\mathrm{Z} 0$ to point $\mathrm{Z} 2$

- $\Delta \mathbf{u}$ - denotes relative velocity of the star regarding the sun

In this way, we have determined the distance and the velocity of the star relative to the solar system.

Now we will discuss the situation shown in Figure (8), when the points $\mathrm{A}, \mathrm{B}, \mathrm{C}$ are collinear and the observer moves uniformly by velocity $\mathbf{v}$.

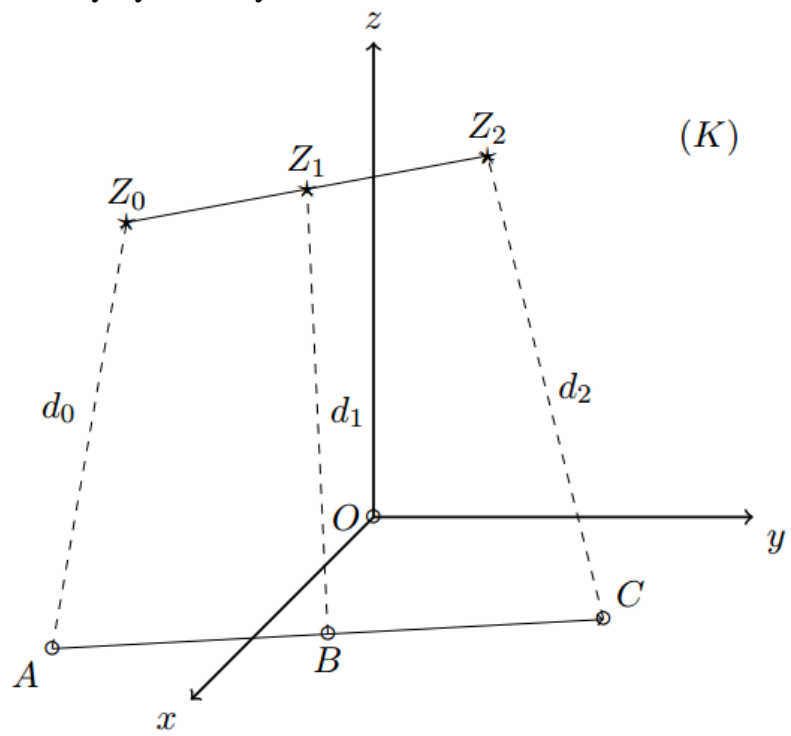

Figure 8: The observer moves uniformly by velocity $\mathbf{v}$ from the point $\mathrm{A}$ to the points $\mathrm{B}$ and $\mathrm{C}$ respectively. There is only a trivial solution.

Referring to Figure (8) we have the following equations:

$$
\begin{array}{r}
\mathbf{v}=\left[v_{x}, v_{y}, v_{z}\right] \\
\mathbf{O C}=\mathbf{O A}+\Delta t_{2} * \mathbf{v} \\
{\left[C_{x}, C_{y}, C_{z}\right]=\left[A_{x}, A_{y}, A_{z}\right]+\Delta t_{2} *\left[v_{x}, v_{y}, v_{z}\right]} \\
=\left[A_{x}+\Delta t_{2} * v_{x}, A_{y}+\Delta t_{2} * v_{y}, A_{y}+\Delta t_{2} * v_{z}\right](90) \\
C_{x}-A_{x}=\Delta t_{2} * v_{x} \\
C_{y}-A_{y}=\Delta t_{2} * v_{y} \\
C_{z}-A_{z}=\Delta t_{2} * v_{z}
\end{array}
$$

In the same way we can get that

$$
\begin{gathered}
\mathbf{O B}=\mathbf{O A}+\Delta t_{1} * \mathbf{v} \\
B_{x}-A_{x}=\Delta t_{1} * v_{x} \\
B_{y}-A_{y}=\Delta t_{1} * v_{y} \\
B_{z}-A_{z}=\Delta t_{1} * v_{z}
\end{gathered}
$$

Combining these equations with (74) and (75) it follows that:

$$
\begin{gathered}
k=\frac{\Delta t_{2}}{\Delta t_{1}} \\
(1-k) * A_{x}+k * B_{x}-C_{x} \\
=k *\left(B_{x}-A_{x}\right)-\left(C_{x}-A_{x}\right): \\
=\frac{\Delta t_{2}}{\Delta t_{1}} * \Delta t_{1} * v_{x}-\Delta t_{2} * v_{x}=0 \\
(1-k) * A_{y}+k * B_{y}-C_{y}=\ldots=0 \\
(1-k) * A_{z}+k * B_{z}-C_{z}=\ldots=0
\end{gathered}
$$

Which leads us to the conclusion that in this case we would have only trivial solutions $\left(d_{1}=d_{2}=d_{3}=0\right)$. We have already assumed that the observed object (star) moves uniformly and if the observer also moves uniformly, which can be said in the following way, if the observed object moves uniformly with respect to the observer then the observer is unable to determine the distance at which the observed object is located.

We will now consider the situation shown in Figure (9). It is obvious that points A, B, C are collinear. But if we define a velocity $\mathbf{v}$ in the following way:

$$
\left(\mathbf{v}=\frac{\mathbf{A B}}{\Delta t_{1}}\right) \Rightarrow\left(\frac{\mathbf{B C}}{\Delta t_{2}}=-\mathbf{v}\right)
$$

Which leads us to the conclusion that observer is not moving uniformly, because velocity $\mathbf{v}$ changes its direction.

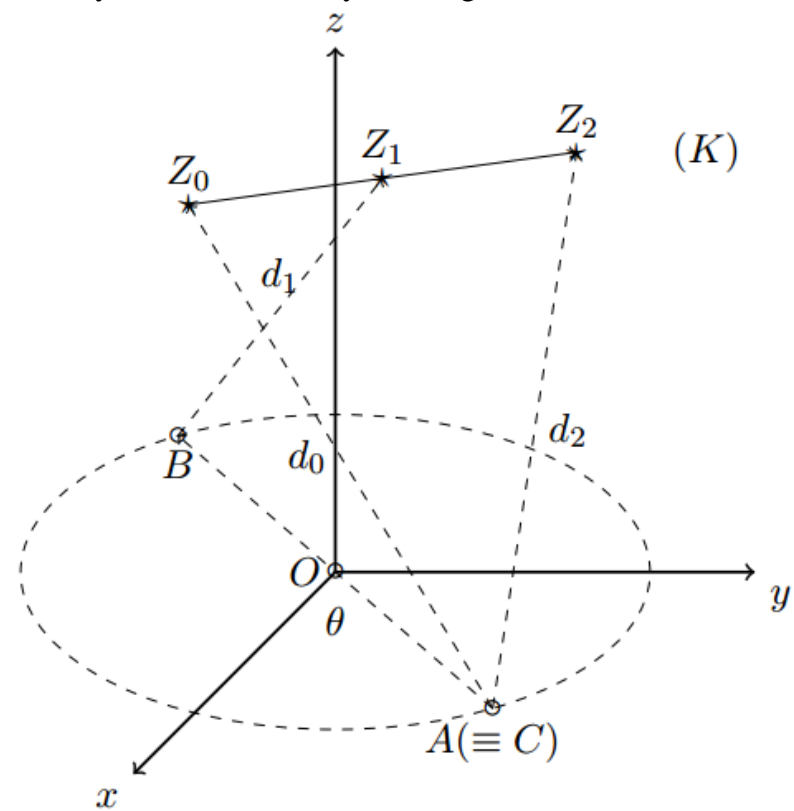

Figure 9: Points A, B, C are collinear, but still there is a nontrivial solution

\section{Volume 8 Issue 9, September 2019}

\section{www.ijsr.net}




\section{International Journal of Science and Research (IJSR)}

ISSN: 2319-7064

ResearchGate Impact Factor (2018): 0.28 | SJIF (2018): 7.426

$$
\begin{gathered}
A_{x}=R * \cos (\theta) \\
A_{y}=R * \sin (\theta) \\
B_{x}=-R * \cos (\theta) \\
B_{y}=-R * \sin (\theta) \\
A_{z}=B_{z}=0 \\
C_{x}=A_{x} \\
C_{y}=A_{y} \\
C_{y}=A_{z} \\
\Delta t_{1}=(0.5+m) * \text { yearsec } \\
\Delta t_{2}=(1+2 m) * \text { yearsec } \\
B_{x}-A_{x}=-2 R \cos (\theta) \\
B_{y}-A_{y}=-2 R \sin (\theta) \\
B_{z}-A_{z}=0 \\
(1-k) * A_{x}+k * B_{x}-C_{x} \\
=k *\left(B_{x}-A_{x}\right)-\left(C_{x}-A_{x}\right)=-2 R \cos (\theta) * k \\
(1-k) * A_{y}+k * B_{y}-C_{y} \\
=k *\left(B_{y}-A_{y}\right)-\left(C_{y}-A_{y}\right)=-2 R \sin (\theta) * k \\
(1-k) * A_{z}+k * B_{z}-C_{z}=0
\end{gathered}
$$

Therefore, equations (82), (83) and (84) have non-trivial solutions.

\section{Testing the proposed methods using data obtained from Gaia's catalogs DR1 and DR2}

Input data are obtained from the Gaia's catalogs [2] (option "Search"). The stars are chosen at random. We can directly obtain the following information:

- RA2015 - Barycentric right ascension of the source in ICRS at the reference epoch J2015.0
- DEC2015 - Barycentric declination of the source in ICRS at the reference epoch $\mathrm{J} 2015.0$

- RA2015.5 - Barycentric right ascension of the source in ICRS at the reference epoch J2015.5

- DEC2015.5 - Barycentric declination of the source in ICRS at the reference epoch J2015.5

- PRX.2015.5 - Absolute stellar parallax of the source at the reference epoch J2015

- PM.RA - Proper motion in right ascension direction, J2015 epoch

- PM.DEC - Proper motion (Declination) J2015.5 epoch

From the obtained data we can derive a right ascension RA2016 and a declination DEC2016 of a star at the reference epoch J 2016.

$$
\begin{aligned}
& R A 2016=R A 2015+\frac{P M . R A}{\cos (D E C 2015)} \\
& D E C 2016=D E C 2015+P M . D E C
\end{aligned}
$$

\begin{tabular}{|c|c|c|c|c|c|}
\hline Gaia source ID & 1996596911406176000 & 1267906854386665088 & 219999464832627584 & 932222445438498944 & 3471190026007380992 \\
\hline RA2015 [degrees] & 345.2287148122942 & 225.83211458394337 & 57.64076463233877 & 124.98562499895499 & 187.33090960579008 \\
\hline DEC2015 [degrees] & 54.38920810592237 & 25.42448994720175 & 36.11354636758435 & 50.884152757844795 & -30.842056520510575 \\
\hline RA2015.5 [degrees] & 345.2286881349424 & 225.83212885046885 & 57.640787372575275 & 124.98564294506525 & 187.33095326939153 \\
\hline DEC2015.5 [degrees] & 54.38919072361569 & 25.424567002148986 & 36.11352384195696 & 50.88412887441873 & -30.842103480719885 \\
\hline Parallax[mas] & 38.45223658078517 & 154.92079559457247 & -0.1736208834797856 & 3.825486423654411 & 24.626355359460785 \\
\hline PM(RA) [mas/year] & -110.82924669719277 & 87.72181780153205 & 148.0180947897536 & 81.4438996869589 & 269.2027204879181 \\
\hline PM(DEC) [mas/year] & -125.75137410621457 & 559.2198769511647 & -162.08048821185827 & -172.20093572981995 & -337.8374762654241 \\
\hline Distance(PRX) $[\mathrm{km}]$ & 8.0247 E14 & $1.991777 \mathrm{E} 14$ & - & $8.0661 \mathrm{E} 15$ & $1.252998 \mathrm{E} 15$ \\
\hline Distance (2CP ) [km] & $8.932 \mathrm{E} 16$ & $3.538 \mathrm{E} 16$ & $9.833 \mathrm{E} 15$ & $2.484 \mathrm{E} 17$ & $1.1 \mathrm{E} 17$ \\
\hline$\Delta \mathbf{u x}(2 \mathrm{CP})[\mathrm{km} / \mathrm{sec}]$ & -2276.67 & -666.83 & 163.94 & 4735.22 & 1416.4 \\
\hline$\Delta \mathbf{u y}(2 \mathrm{CP})[\mathrm{km} / \mathrm{sec}]$ & -328.02 & 3004.14 & -288.21 & -5515.41 & 7174.67 \\
\hline
\end{tabular}

All these coordinates are given with respect to the equatorial coordinate system. In order to apply Equation (41), it is necessary to transform these coordinates into ecliptic coordinate system $(\mathrm{K})$. The transformation matrix from the equatorial to the ecliptic coordinate system is given in [1].

Mow we are now able to find the distance and transverse velocity of the star relative to the sun.

Distance(2CP ) - Derived from the Equation (41)

$$
\begin{array}{r}
d=\text { Distance }(2 C P) \\
\Delta \mathbf{u}_{x}=\frac{d * \tan (\text { PM.LONG })}{\text { yearsec }} \\
\Delta \mathbf{u}_{y}=\frac{d * \tan (\text { PM.LAT })}{\text { yearsec }}
\end{array}
$$

Where PM.LONG and PM.LAT denote components for proper motion of the star in the ecliptic coordinate system (K).

The results are summarized in the two following tables, which are copied from [1], except for the last three rows.

Table 1: Distance between the Earth and the star and star velocity calculated on the basis of the data obtained from the Gaia's catalogs 


\section{International Journal of Science and Research (IJSR) \\ ISSN: 2319-7064 \\ ResearchGate Impact Factor (2018): 0.28 | SJIF (2018): 7.426}

Table 2: Distance between the Earth and the star and star velocity calculated on the basis of the data obtained from the Gaia's catalogs

\begin{tabular}{|c|c|c|c|c|c|}
\hline Gaia source ID & 3968823147582581504 & 3506524893749579648 & 2392703946771797248 & 1854353916756790400 & 3945681073517688960 \\
\hline RA2015 [degrees] & 164.37525525103945 & 200.09638529784812 & 350.6790229293687 & 321.88591137228934 & 185.63609385569734 \\
\hline DEC2015 [degrees] & 13.653681201911555 & -20.06343186628493 & -19.691300195639307 & 34.0238623055378 & 16.357596564626736 \\
\hline RA2015.5 [degrees] & 164.37528560226255 & 200.09640104426754 & 350.67898899365645 & 321.8858646334176 & 185.63607166653534 \\
\hline DEC2015.5 [degrees] & 13.653657150053572 & -20.063447680691795 & -19.6913255777869 & 34.02383684263199 & 16.3575826008146 \\
\hline Parallax[mas] & 20.911752813322053 & 11.496169556523695 & 23.25135907901302 & 38.45223658078517 & 15.40129228431169 \\
\hline PM(RA) [mas/year] & 211.58298658446174 & 105.17501228068048 & -229.9247639971531 & -280.0377248573189 & -153.87591844804413 \\
\hline PM(DEC) [mas/year] & -172.99188421088598 & -113.83221442757147 & -183.05964366990693 & -182.44201795818088 & -100.88155554235291 \\
\hline Distance(PRX) [km] & $1.47557 \mathrm{E} 15$ & $2.684 \mathrm{E} 15$ & $1.327 \mathrm{E} 15$ & $8.0247 \mathrm{E} 14$ & $2.0035186 \mathrm{E} 15$ \\
\hline Distance $((2 \mathrm{CP}))[\mathrm{km}]$ & $1.071 \mathrm{E} 17$ & $9.456 \mathrm{E} 16$ & $2.014 \mathrm{E} 17$ & 6.7924 E16 & $6.613589 \mathrm{E} 16$ \\
\hline$\Delta \mathbf{u x}(2 \mathrm{CP})[\mathrm{km} / \mathrm{sec}]$ & 4309.64 & 699.89 & -4056.81 & -3465.84 & -988.09 \\
\hline$\Delta \mathbf{u y}(2 \mathrm{CP})[\mathrm{km} / \mathrm{sec}]$ & -1284.22 & 2140.19 & 8142.27 & -389.52 & -1580.73 \\
\hline
\end{tabular}

From the data presented in Table (1) and Table (2), we can conclude that there are huge differences between the results obtained by different methods. It can also be observed that the results obtained by the parallax method are quite different compared to the other two methods. The following table shows the data for cases where parallax has a large negative value or is very close to zero.

Table 3: Distances between the Earth and some stars with negative or very small parallaxes

\begin{tabular}{|c|c|c|c|c|c|}
\hline Gaia source ID & 4062375820315455104 & 1820917428872070400 & 4268038405111900160 & 2183214172431040384 & 4079164984890216448 \\
\hline RA2015 [degrees] & 269.6730431886717 & 296.2907746753095 & 287.5377088230294 & 308.70236144293267 & 279.96399315473826 \\
\hline DEC2015 [degrees] & -28.972101813547678 & 16.947768042205972 & 2.2771464168140048 & 53.63920570113808 & -22.817303658487507 \\
\hline RA2015.5 [degrees] & 269.6732013722213 & 296.2907040973579 & 287.5378054418249 & 308.70236161991096 & 279.96399432106153 \\
\hline DEC2015.5 [degrees] & -28.97209003428289 & 16.94766502076807 & 2.2771272336601984 & 53.639205662074886 & -22.817303631496998 \\
\hline Parallax[mas] & -630.801289322115 & -744.21514648868885 & -452.15706867060175 & -0.004070265025264887 & 0.03320771710519638 \\
\hline PM(RA) [mas/year] & 757.5128164066781 & 125.69240316245498 & 47.60957378935429 & -1.4155986603218278 & 25.66026691924152 \\
\hline PM(DEC) [mas/year] & -566.5674247635498 & 39.2940055799761 & 23.943751870781398 & -0.6699908476680323 & 20.000714576902016 \\
\hline Distance(PRX) $[\mathrm{km}]$ & - & - & - & - & $9.292 \mathrm{E} 17$ \\
\hline Distance(3P) $[\mathrm{km}]$ & $3.80 \mathrm{E} 13$ & $2.033 \mathrm{E} 16$ & $9.88 \mathrm{E} 13$ & $1.37 \mathrm{E} 17$ & $9.128 \mathrm{E} 14$ \\
\hline Distance((2CP)) $[\mathrm{km}]$ & $1.50 \mathrm{E} 14$ & $1.20 \mathrm{E} 14$ & $2.0846 \mathrm{E} 14$ & $1.03 \mathrm{E} 17$ & $4.192 \mathrm{E} 15$ \\
\hline$\Delta \mathbf{u x}(2 \mathrm{CP})[\mathrm{km} / \mathrm{sec}]$ & 17.44 & 2.42 & 1.61 & -23.95 & 14.91 \\
\hline$\Delta \mathbf{u}(2 \mathrm{CP})[\mathrm{km} / \mathrm{sec}]$ & 13.15 & 0.19 & 0.56 & 6.37 & -14.20 \\
\hline
\end{tabular}

From the results shown in Table (3) we can make conclusion that some of the stars with large negative or very small parallaxes are relatively close to the solar system. Which only confirms our assumption that the distance cannot be determined from the parallax angle.

\section{Conclusion}

In fact, we will not make any definitive conclusions regarding the proposed methods. They need to be further tested with a far greater number of samples. Only in this way will we be able to detect any errors in the proposed procedures and input data. Then we will be able to make comparisons between the results and make an assessment of their accuracy.

\section{References}

[1] Cojanovic M. (2019) Stellar Distance and Velocity Journal of Applied Mathematics and Physics, 7, 181209. https://doi.org/10.4236/jamp.2019.71016

[2] (2018) Gaia Archive https://gea.esac.esa.int/archive/

[3] Wikipedia contributors. "International Celestial Reference System." Wikipedia, The Free Encyclopedia. Wikipedia, The Free Encyclopedia, 16 Apr. 2019. Web. 7 Jul. 2019 